\title{
Bifurcation Dynamics: Theory, Methods, and Applications
}

\author{
Jocirei D. Ferreira • Ronaldo A. Garcia • \\ V. Sree Hari Rao
}

Published online: 22 August 2012

(c) Foundation for Scientific Research and Technological Innovation 2012

Bifurcation theory is a subject with classical mathematical origin. The modern development of this subject dates back to the pioneering work of Poincaré, and over the past four decades this theory has witnessed rapid developments with new ideas and methods from dynamical systems theory, singularity theory and many others.

This subject relates to the mathematical study of possible changes in the qualitative or topological structure of parameter-dependent systems (families). Bifurcations occur in both continuous systems (described by ODEs, DDEs or PDEs), and discrete systems (described by maps).

It is useful to place the subject of bifurcations in two principal classes such as local and global. Local bifurcations occur when variations in parameters cause changes in the stability of equilibrium (or fixed point). The topological changes in the phase portrait of the system may be confined to arbitrarily small neighborhoods of the bifurcating fixed points by moving the bifurcation parameter close to the bifurcation point (hence 'local'). It is worth to recall that local bifurcations can produce changes in global behavior and that in the mathematical analysis of local bifurcations problems it is necessary to understand global dynamics, i.e., "punctual singularities can be a concentration of global information". Global bifurcations occur when 'larger' invariant sets such as periodic orbits collide with equilibria. This brings changes in the topology of the trajectories in the phase space which cannot be confined to

\footnotetext{
J. D. Ferreira $(\varangle)$

Institute of Exact and Earth Science, Federal University of Mato Grosso, Rodovia BR-070, km 5, Barra do Garças, MT CEP 78600-000, Brazil

e-mail: jocirei@ufmt.br

\section{R. A. Garcia}

Instituto de Mathemática e Estatística, Universidade Federal de Goiás, Caixa Postal 131, Goiânia, GO CEP 74001-970, Brazil

e-mail: ragarcia@mat.ufg.br

\section{Sree Hari Rao}

Institute for Development and Research in Banking Technology, Castle Hills, Road No. 1, Masab Tank, Hyderabad, Andra Pradesh 500 057, India

e-mail:vshrao@idrbt.ac.in
} 
a small neighborhood of a point, as in the case of local bifurcations. In fact, the changes in the topology extend out to an arbitrarily large distance (hence 'global'). There are several important application areas of bifurcation theory and this theory has also been applied in the study of several theoretical examples which are difficult to access experimentally.

In recent years many types of bifurcations of flow and maps have been studied and classified including saddle node, Hopf, umbilic, zip, homoclinic tangencies, period doubling and cusp bifurcations. It is our belief that in the years to come the bifurcation theory plays a more active role in various application domains of Science and Technology. Our thoughts and efforts along these lines have culminated in the organization of the current research as a special issue of thoroughly reviewed articles that are of interest to the scientific community working in these areas.

In response to our call for papers eight papers that met the high standards of the journal were approved for publication in the special issue after two full rounds of reviewing.

It is our pleasure to thank the reviewers for their critical reviews which have contributed immensely to the decision making process. 